\title{
GENERATING COLLABORATIVE WORK PROCESSES
}

\author{
I.T. Hawryszkiewycz \\ Department of Information Systems \\ University of Technology, Sydney \\ igorh@it.uts.edu.au
}

ABSTRACT: The paper describes ways to support collaboration in business processes. Collaborative processes are different from predefined processes in the sense that they can change dynamically as the situation emerges. Such changes can be time consuming as they require users to continually adapt the system to changing contexts. The solution proposed here to support process evolution is to provide generic work objects and use software agents to assist users to dynamically change the process by quickly adding or changing work objects. The paper outlines a way of describing work processes in terms of generic work objects. The structure of the generic work objects is based on a metamodel, which provides the fundamental concepts to define generic objects. A prototype implementation is then described..

KEYWORDS: Process definition, Collaboration, Workspaces, Software agents

\section{INTRODUCTION}

Many business processes now support collaboration in applications such as distributed project teams, software development teams (Carmel, 1999), design teams, planning and evaluation teams, or client support teams. Because of their distributed nature, such processes increasingly rely on the InterNet to support collaboration. However, studies (Cummings, 2002) have found that collaboration on the InterNet usually does not go beyond simple communicative acts such as exchange of documents. Often as found by Duchenaut and Belotti (2001), users develop their own personal support systems, usually on their private systems. Users must then continually integrate them into collaborative activities as needed. Such integration can be quite time consuming to avoid inconsistencies between individual records on personal systems and the enterprise context, as well as to transfer user contributions into the context.

Furthermore collaborative processes tend to emerge and evolve as they proceed. Such evolution calls for constant changes to services to be provided to process participants. This paper suggests a way to provide customizable work objects that can be easily configured to different collaborative activities within the enterprise context. Figure 1 illustrates the idea. Here there is a library of work objects. These are combined into one work process to meet a given work objective.

To do this the paper proposes a way to define work objects that are generic in nature and widely applicable. These objects can be combined to accomplish an objective within the given context. The paper provides a metamodel for this purpose. The metamodel provides the concepts and structure needed to define the generic work objects. The metamodel combines both process and social aspects to represent collaborative activities.

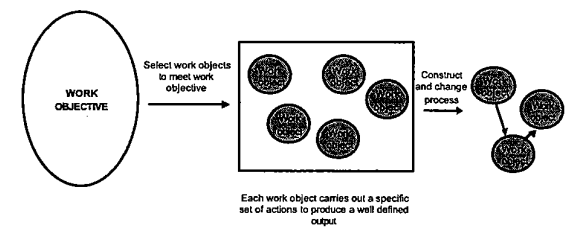

Figure 1 - Creating a Process

The other key requirements are agents to combine the work objects into work processes. The process for doing so is shown in Figure 2. It shows 
two kinds of agents. The first are agents that assist users to combine such work objects into a process Such agents must support organizational processes that produce well-defined documents, such as market reports, which must be developed as part of the process. The second are agents that manage and change the concrete processes.

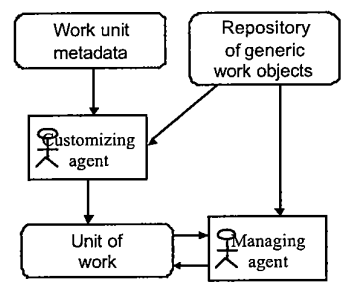

Figure 2- Generic and concrete units of work

The goal pof the paper is to describe a way to define such work units. The paper first defines the metamodel, which provides the foundation for defining the work objects in collaborative environments. The paper then describes some work objects..

\section{SEMANTICS TO DESCRIBE WORK OBJECTS}

The work objects proposed here are generic in the sense that they can be adapted to many processes. The metamodel described here combines process responsibility as well as social networking. It is shown in Figure 1 and is an extension of a model described earlier (Hawryszkiewycz, 2005). The metamodel covers the process parts and has evolved over a number of years. It builds on concepts from earlier systems such as Conversation Builde (Kaplan, 1992) or Oval (Malone, 1992) and has been verified through a variety of applications that include business networking (Hawryszkiewycz, 1996), and strategic planning (Hawryszkiewycz, 1997). This paper extends the process model to incorporate the social awareness network within the activity structure. The combined model shown in Figure 1 includes two levels. One is the process level, wh ich defines the formal roles and activities in the process. The process structure represents the arrangements in place for collaboration. It shows the documents available in the system and their use in different activities. The second is the socia awareness network, which supports user socia interactions. These in many cases can change as collaboration evolves.

\subsection{The process level}

In the process level, the rectangular boxes represent concepts whereas lines between the oval shapes are relationships between the concepts. Figure 3 also groups the process structure concepts into three parts, namely:

- The work activities, which are modeled as activities and actions. These actions usually refer to or change artifacts. An activity can include many actions, which in turn can use many artifacts. Responsibilities for such actions are assigned to designed roles.

- The people and how they are organized into groups. .Any person or participant can be part of a number of groups, and each group can have any number of participants. Groups can include groupings into departments or other units. The groups can then assume roles with defined responsibilities in organizational activities. This part of the metamodel provides ways to combine work-actions into activities with members of groups assigned responsibilities through roles for those workitems

- Workflows, which are supported by associating events with roles. People associated with these roles can initiate completion events, which in turn trigger initiation events that notify roles to carry out their tasks.

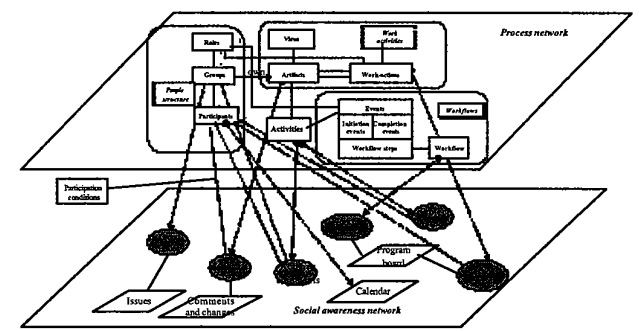

Figure 3 - A Metamodel for Defining Process Communication Patterns 
These three kinds of concepts are essential for modeling business applications. Most business processes follow a workflow, they involve organizational activities and they require social interactions to share knowledge.

\subsection{The Social Level}

This paper examines ways to extend the process network to include social aspects. It places a social awareness network in parallel with structured forma activities. The social network is composed of discourses that emerge as collaboration evolves. These are represented by oval shapes. Each discourse is an oval shaped box. In addition there is a collaborative database where such interactions are recorded. Usually each such database is created by one component of the process structure. This can include issues boards, discussions, or various comments on progress. Eventually the kind of discourse would be supported by the most appropriate technology (Barton, 2005). A typical definition of a discourse is:

Discourse (<discourse-type>, <initiated by>, $<$ associated collaborative database $>$, \{participants, rules\})
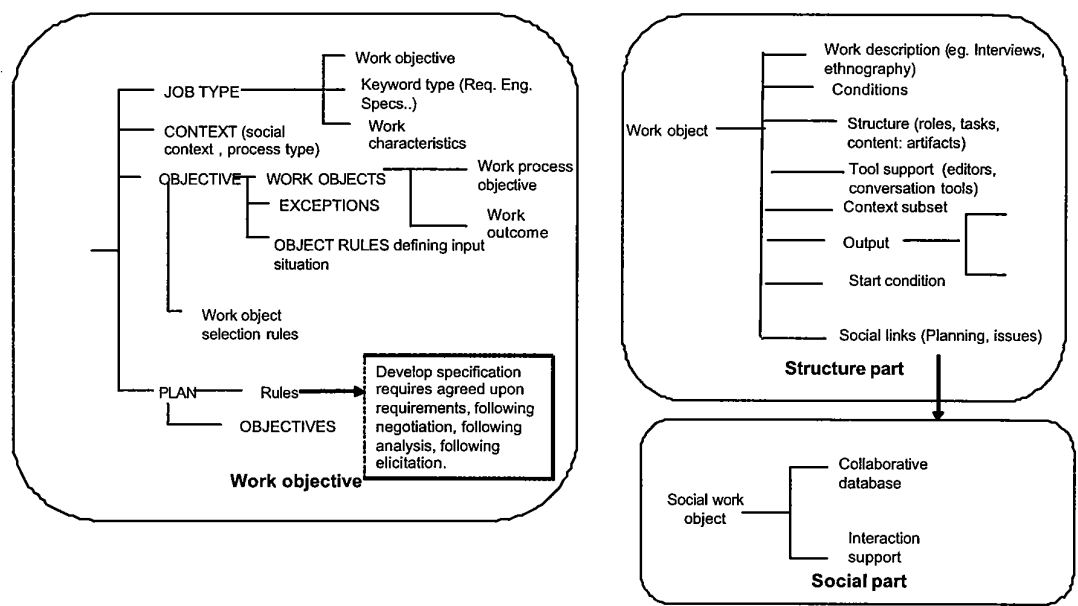

Participation in discourses is defined by rules with rules chosen to ensure a desired level of collaboration. As an example we could have:

<group-membership-issues> (issues and comments, 'project team-1', 'participation rules', all members of team)

This defines discussions about adding members to project team-1. It defines that all existing members of the team can participate and issues and comments raised are kept in a database called 'participation rules'.

\subsection{Level Integration}

The relationship between the process and social levels is defined by the dotted lines in Figure 3. The dotted line that starts with the circle shows the concept that initiates a discourse. Other dotted lines show the participants and groups that participate in the discourse.

Figure 4-Work descriptions 


\subsection{Structure of work objects}

The semantic model provides the guidelines for specifying work objects. Figure 4 is a structure of work object, which is composed of elements that correspond to the process level concepts in the metamodel shown in Figure 3.

The work objective here is to collect requirements in a software development project. The plan follows the normal process of collecting information, resolving any conflicts by negotiation and then developing a specification. Only one work object is shown - collecting information. The collecting information work object, which is chosen, will depend on the social context.

The work instance includes a plan to choose work objects depending on the social context and as specified by the selection rules. Thus it is possible to choose an object that supports interviews, or an ethnographic approach.

Each work object can have any number of links to social objects as often occurs in collaborative activities. These can be discussions, blogs or wikis depending on the type of relationships to be maintained (Barton, 2005).

\section{WORK OBJECTS FOR COLLABORATION}

The work objects commonly found in collaborative work include:

e-portfolio - Supports working on an artefact by number of people. It supports a collection of artefacts developed by a number of people. Different responsibilities are assigned in the portfolio. Examples include - education with teacher and student responsibilities. Strategic documents with planning and expert responsibilities or paper preparation with author and reviewer responsibilities. The parameters of this e-portfolio will be document names, roles and role responsibilities for each document.

The e-portfolio can also be defined grammatically as follows:

e-Portfolio: portfolio-name;

Work-goal: (Text with keywords)

Work-roles: $+\{<$ roleContent: work-content: $+\{<$ artifact-name $>\}$; services: $+\{<$ service-name $>\}$ +actions: $\{$ artifact: + artifact-name $\}\}$, \{services: $+\{<$ service-name $>\}\}$ $+\{$ action: $\{+\{<$ role name $>$, services: + \{service-name $\}$ information: $+\{$ artifact-name $\}$;

There are also constraints and permissions, as for example, role permissions to access information, and what kind of access is permitted. The kinds of semantics include:

Create-e-portfolio

Invite people to take up a role

Add artefacts to the e-portfolio,

Alert people of actions taken by others in the eportfolio

Setup services to support actions in the eportfolio.

The e-portfolio in this case can be seen as collaboration in the small being carried out within a larger framework. The issues then are how to subdivide a process into e-portfolios while maintaining links to the entire context.

Workflow instance - To arrange work actions associated with an activity. Here a workflow is defined in terms of events, which are assigned to roles. A completion event initiated by one role can result in an initiation event for some other role. The process can change dynamically by adding new events dynamically.

Group management - managing a group of people, which may be an organizational unit or people with common interests. Usually requires support for sharing information, managing group changes and maintaining group memory in general.

Team formation - requires support for keeping track of activities and responsibilities of individual team members. Important aspects are new members joining teams, resolution of issues and distributing work between team members, including negotiation for assigning and carrying out tasks..

Program and issues boards. There are a number of advantages of using such higher level concepts in collaborative systems. One is to provide a social construct that can be easily understood. Another is that interactions as particularly suitable as a way of integrating processes. It provides such a basis ranging from predefined 
processes to emerging processes that include supporting mobility in the workforce. It can be used as the basis for supporting communication beyond the simple exchange of messages to supporting more goal oriented communication that integrates a number of messages into the one interaction. It however sees that support must be provided to manage such interactions and suggests agents as suitable for this purpose. Conceptually it can be viewed as a composite object [5] that can be represented in terms of modeling concepts such as entities or relationships.

Low collaboration levels usually require e-portfolios and perhaps group management. Higher levels of collaboration will require engagements such as team formation or workflow instance.

An example of a process defined in terms of generic objects is shown in Figure 5. It starts with developing an e-portfolio on requirements identified though interviews and other conversations. It then continues with a negotiation to set priorities. The las step is an e-portfolio that results in a specification. Part of the specification is an e-portfolio of system models.

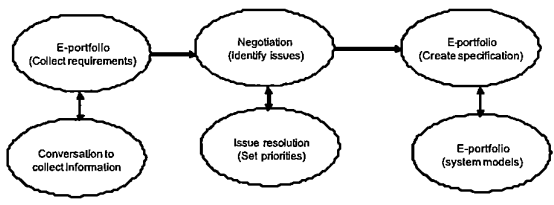

Figure 5 - A Work Instance

\section{AN IMPLEMENTATION}

We are developing prototype generic agents for our workspace system, LiveNet. Figure 6 illustrates a typical workspace that supports the capturing user requirements. This is the top level workspace that describes the work process.
It lists the three work-activities in the selected plan, namely, elicitation, modeling and specification development. It also shows the work context including the system description and other organizational information.

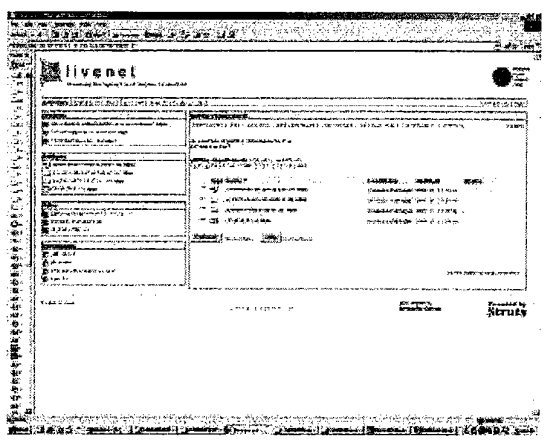

Figure 6-A workspace for determining user requirements

\section{Creating work activities}

Figure 7 illustrates one of the work objects that make up requirements elicitation. It now shows the participants in the process and their roles - user, analyst and manager. It also shows a social object, namely a blog space, which is used to clarify various issues identified during interviews. Each analyst in this workspace has a view that contains a collection of interview reports. These can be accessed by other analysts in the team for comment. Furthermore each analyst can construct a blog to collect comments on their activities and outputs.

The participants of the workspaces must now carry out the actions defined for the activity. 


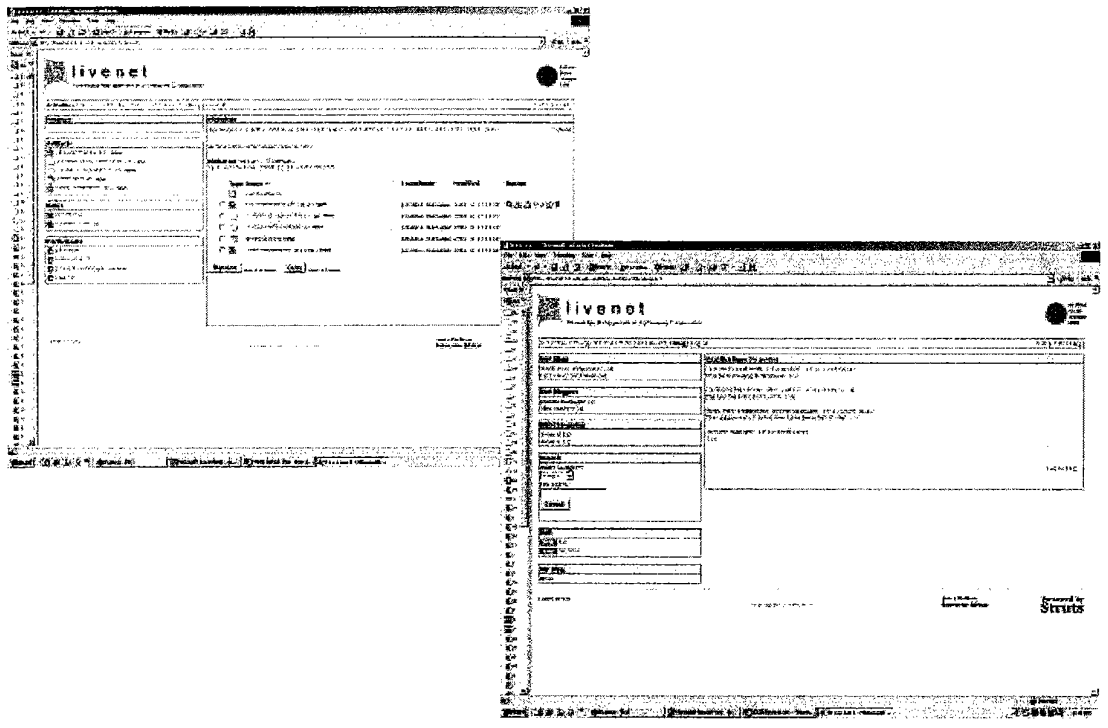

Figure 7 - Work object for eliciting requirements

\section{AGENTS FOR CUSTOMIZING WORKSPACES}

Our goal as shown in Figure 2 is to develop agents to construct workspaces to support collaborative processes

Figure 8 shows the principal activities of the customizing agents.

Customizing agents predominantly match open parameters to user preferences.

\section{Subgoal:setup Custom-UOW}

If work-metadata(work-description) matches UOW(work-description) then create custom-UOL from UOL; add UOW(work-content) to CustomUOW(work-content); if UOW(work-output) matches work-output (work-output-description) then add work-metadata(work-output(name): UOW(work-output);

Subgoal: setup custom-work-plan
If sum of work-plan(activity-objectives) includes all keywords in Custom-UOL(work-description) and

work-plan (plan-type) matches CustomUOL(unit-plan-options)

then create custom-work plan from work plan.

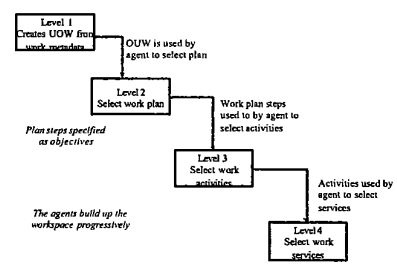

Figure 8 - Constructing units of work

\subsection{Managing Agents}

The general rules here are that each work object has an associated agent. The type of agent corresponds to the kind work object. Our earlier 
work (Hawryszkiewycz, Lin, 2003) defined a set of generic agents for managing collaborative processes. These were based on a metamodel of collaboration and included:

\subsection{Defining the agents}

At a more detailed level, we use the usual reasoning model of agents shown in Figure 6 and implement it using the three layer architecture Müller, 1996) chosen from a number of alternative architectures (Woodridge, 1999). Agents are used to achieve goals using plans defined by agent users. A plan is composed of event-condition-action rules, each of which specifies the actions to be executed when condition is true.

An example is support for personalized learning (Pan, Hawryszkiewycz, 2006) for creating workspace created for learning.

An example is shown in Figure 9. It shows a sequence of workspaces generated by agents to support a learning work process. It first identifies a learner goal and then suggests a plan to be followed to satisfy the learning goal. It then displays the selected plan. Once accepted the agents will generate workspaces to support the learning activities of the plan.

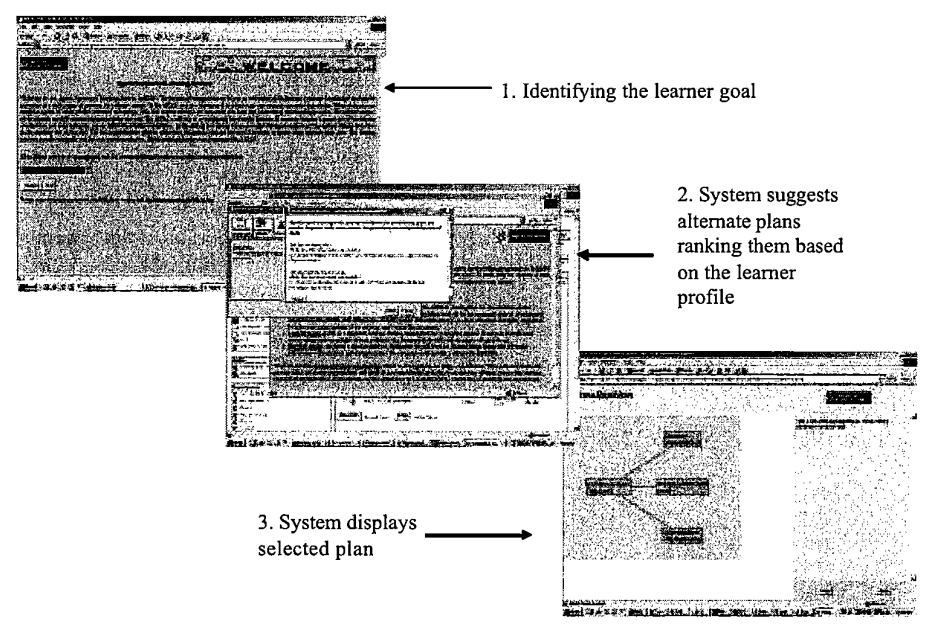

Figure 9 - Evolving workspaces for learning

\section{SUMMARY}

This paper described the development of specifying collaborative processes that are supported by software agents. It described a generic set of software agents. The paper then describes a way of customizing generic work-units into work processes. It then proposed a set of customizing agents to construct concrete processes and managing agents to support these processes.

\section{ACKNOWLEDGEMENTS}

The work described here was supported by an ARC Discovery grant. The contribution of Aizhong $\mathrm{Lin}$ in defining the agent architecture is also acknowledged. 


\section{REFERENCES}

Barton, M.D. (2005): "The future of rational-critica debate in online public spheres" Computers and Composition 22, pp. 177-190, Elsevier Press.

Belanger, F. (1999): "Communication Patterns in Distributed Work Groups: A network Analysis" IEEE Transactions on Professional Communication, Vol. 42, No. 2, December 1999, pp261-275.Carmel E. (1999): "Global Software Teams" Prentice-Hall, Upper Saddle River

Carmel, E. (1999): "Global Software Teams" PrenticeHall, Upper Saddle River.

Cummings, J.N., Butler, B. and Kraut, R. (2002): "The Quality of OnLine Social Relationships" Communications of the ACM, Vol. 45, No. 1, July, 2002, pp. 103-111.

Ducheneaut, N. and Bellotti, V. (2001): "E-mail as Habitat' Interactions, September-October, 2001, pp 30-38

Hansen, M.T., Nohria, N. and Tierney, T. (1999): "Whats your Strategy for Managing Knowledge" Harvard Business Review, March-April, 1999, pp. 106-116.

Hattori, F., Ohguro, T., Yokoo, M., Matsubara, S. and Yoshida, S. (1999): "Socialware: Multiagent Systems for Supporting Network Communities", Communications of the ACM, March, 1999, pp. 5559.

Hawryszkiewycz, I.T (2005): "A Metamodel for Modeling Collaborative Systems" Journal of Computer Information Systems, Vol. XLV, Number 3, Spring 2005, pp. 63-72.
Hawryszkiewycz, I.T. (June, 1996):" Providing Compute Services For Business Networks" Proceedings of the Ninth International Conference on EDI-IOS, ISBN961-232-000-4, Bled, June, 1996, pp. 398-411.

Hawryszkiewycz, I.T., Steele, R. (2005): “A Framework for Integrating Mobility into Collaborative Business Processes" Proceeding of the Conference on Mobile Business, Sydney, July, 2005, pp. 89-93.

Hawryszkiewycz, I.T. and Lin, A.(2003): "Process Knowledge Support for Emergent Processes" Proceedings of the Second IASTED International Conference on Information and Knowledge Management, Scottsdale, Arizona, November, 2003, pp. 83-87.

Kaplan, S.M. , Tolone, W.J., Bogia, D.P. and Bignoli, C. (1992): "Flexible, Active Support for Collaborative Work with ConversationBuilder" Proceedings of the CSCW'92 Conference, November 1992, Toronto, pp. 378-385.

LiveNet: http://livenet4.it.uts.edu.au

Malone, T.W. and Fry, C. (1992): "Experiments with Oval: A radically Tailroable Tool for Collaborative Work" Proceedings of the CSCW' 2 Conference, November 1992, Toronto, pp. 289-297.

LiveNet, http://livenet4.it.uts.edu.au

Müller J. P. (1996). The design of Intelligent Agents. Springer Verlag. 1996. Pp. 7-44

Pan, W., Hawryszkiewycz, I.T. (2006): "Assisting Learners to Dynamically Adjust Learning Processes through Software Agents" International Journal of through Software Agents" International Journal of
Intelligent Information Technologies, Vol. 2, No. 2 Intelligent Information Tec.

Wooldridge, M. (1999): "Intelligent Agents" in Chapter 1 "Computational Organization Theory" by KM Carley \& L Gasser in "Multiagent Systems" Gerhard Weiss (Ed) MIT Press - 1999 